\title{
A conducting composite of polypyrrole II. As a gas sensor
}

\author{
Fatma Selampinar ${ }^{a}$, Levent Toppare ${ }^{a, *}$, Ural Akbulut ${ }^{a}$, Talat Yalçin ${ }^{a}$, Şefik Süzer ${ }^{b}$ \\ ${ }^{a}$ Department of Chemistry, Middle East Technical University, 06531 Ankara, Turkey \\ ${ }^{b}$ Department of Chemistry, Bilkent University, 06533 Ankara, Turkey
}

Received 10 February 1994; revised 11 August 1994; accepted 2 September 1994

\begin{abstract}
Pure polypyrrole (PPy) and polypyrrole-polyamide (PPy-PA) composite films were synthesized electrochemically. The gassensing ability was investigated for both pure PPy and PPy-PA films. The composite films'response to several gases are better defined and reproducible compared to pristine conducting polymer. Electrochemical behaviour of PPy and PPy-PA electrodes in the presence of pyrrole and pyrrole-free medium is investigated via cyclic voltammetry. Mass spectrometry studies strictly reveal that the composite is completely different to a mechanical mixture. This phenomenon is discussed in comparison to polyaniline-polycarbonate composite.
\end{abstract}

Keywords: Composite; Polypyrrole; Gas sensor

\section{Introduction}

Electrically conducting polymers are an extremely interesting class of materials that have gained popularity in the last decade and have been synthesized by chemical and electrochemical methods covering polyacetylene $[1,2]$, polypyrrole (PPy) [3], polythiophene [4], polyaniline (PAn) $[5,6]$. They can be used in energy storage [7-9], solar energy conversion $[10,11]$ and electrochromic display devices [12].

Today, protection of the environment and persons is more important than before. For that reason there is a need for improved or new sensors for measuring both physical and chemical changes, such as humidity, temperature, pressure and chemical species. Nowadays there is a great interest in making conducting polymer sensors. Several organic materials have been shown to exhibit resistivity changes when exposed to a variety of gases [13-17]. It is convenient to make them as thin films laid down over interdigitated electrodes. In this case, the electrochemical synthesis of doped conducting polymer is the most convenient. They can be used to sense gases and vapours by following the change in resistance of the polymer. The detailed mechanism of this response has not yet been understood. However, preliminary studies have shown that they exhibit fast,

\footnotetext{
* Corresponding author.
}

reversible responses at room temperature. They have many advantages over other sensors. First, a wide variety of polymers are available. Secondly, they are readily grown by electrochemical polymerization of the monomer. Thirdly, they operate at room temperature. One disadvantage is the lack of specificity.

The gas-sensing properties of PPy were demonstrated using a sensing element consisting of pyrrole (Py) blackimpregnated filter paper. Miasik et al. [14] prepared a sensor utilizing a black PPy layer laid down on an interdigitated gold electrode, screen printed on alumina substrate. This was used as an anode and a gold wire as the cathode. Almost immediately the PPy film became visible as a black film on the gold electrodes of the sensor. The fine electrode spacing allowed growth of the polymer film across the interdigitated electrode. The response of electrochemically prepared PPy to $\mathrm{NO}_{2}, \mathrm{H}_{2} \mathrm{~S}$ and $\mathrm{NH}_{3}$ was demonstrated. Later, studies on the production of PPy-based sensors were increased [15-18].

It is known that PPy is a p-type semiconductor. The exposure of electrophilic gases such as $\mathrm{NO}_{2}$ to $\mathrm{PPy}$ causes an increase in conductance. Electron-donating gases such as $\mathrm{NH}_{3}$, if adsorbed, have an opposite effect. Hence, there is a resistance increase of conducting PPy upon exposure to $\mathrm{NH}_{3}$.

We proposed that the hopping process is affected via grafting or copolymerization of the conducting poly- 


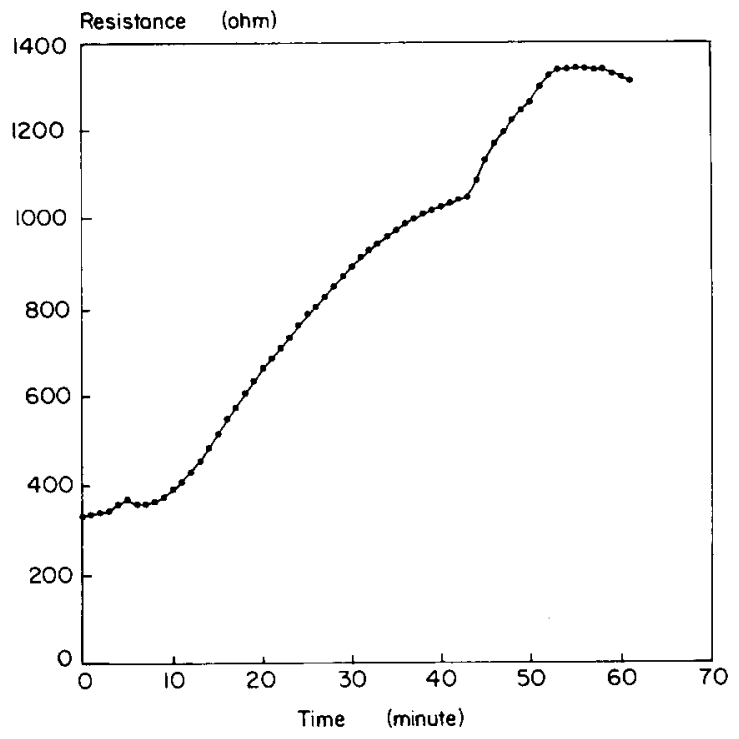

Fig. 1. Resistance changes at room temperature for the pure PPy film sensor to $\mathrm{NH}_{3}$. Flow rate, $100 \mathrm{ml} / \mathrm{min}$.

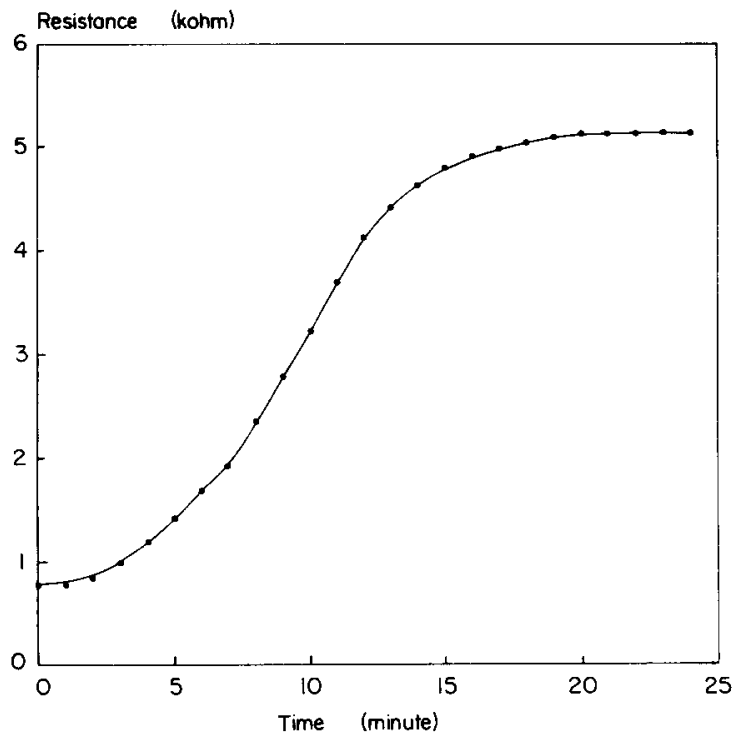

Fig. 2. Resistance changes at room temperature for the PPy-PA film sensor to $\mathrm{NH}_{3}$. Flow rate, $100 \mathrm{ml} / \mathrm{min}$.

mer. That is, if several sites can be added to the conducting polymer to enhance the hopping process the response to such oxidizing or reducing gases can be more pronounced, at least in terms of reproducibility and reversibility $[6,13]$. Thus, the use of such a conducting polymer may reveal several properties with respect to pristine conducting polymer in terms of gas sensing.

Here we report the response of polypyrrole-polyamide (PPy-PA) and PPy films to $\mathrm{NH}_{3}$ and $\mathrm{CO}_{2}$. Electroactivity of the electrolytic films compared to pure PPy was also studied with cyclic voltammetry. In a previous paper we mentioned several properties of a polycarbonate-polyaniline (PC-PAn) film in com-
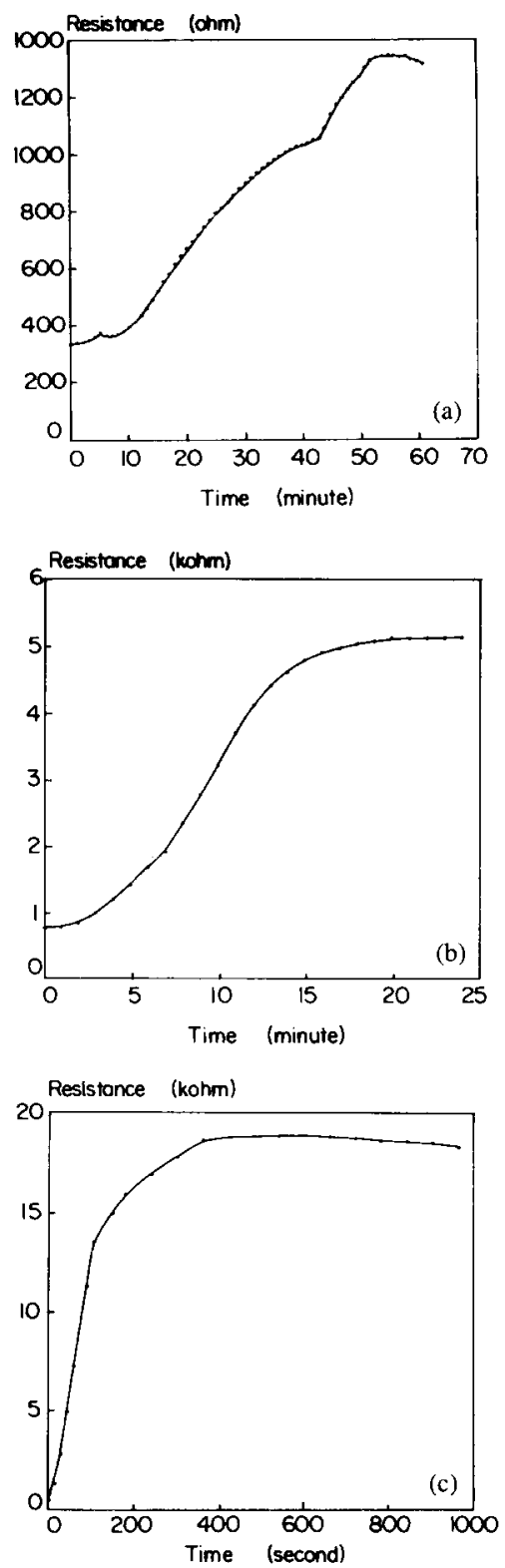

Fig. 3. Resistance changes at room temperature for (a) $100 \% \mathrm{PPy}$, (b) $78 \%$ PPy and (c) $40 \%$ PPy films to $\mathrm{NH}_{3}$. Flow rate, $100 \mathrm{ml} /$ min.

parison to PAn synthesized in the same manner [13]. Similarities between the two composite films were discussed. The synthesis and characterization of PPy-PA film were given in an earlier report [19]. The electrolytic film in the gas-sensing processes was $60 \%$ in PPy and it represents an intimate mixture of the two polymers which yields evidence of a chemical bonding between the two polymers [19].

\section{Experimental}

Polymerization of Py was carried out in a threecompartment cell containing $\mathrm{CH}_{3} \mathrm{CN}-\mathrm{TBAFB}$ and the 

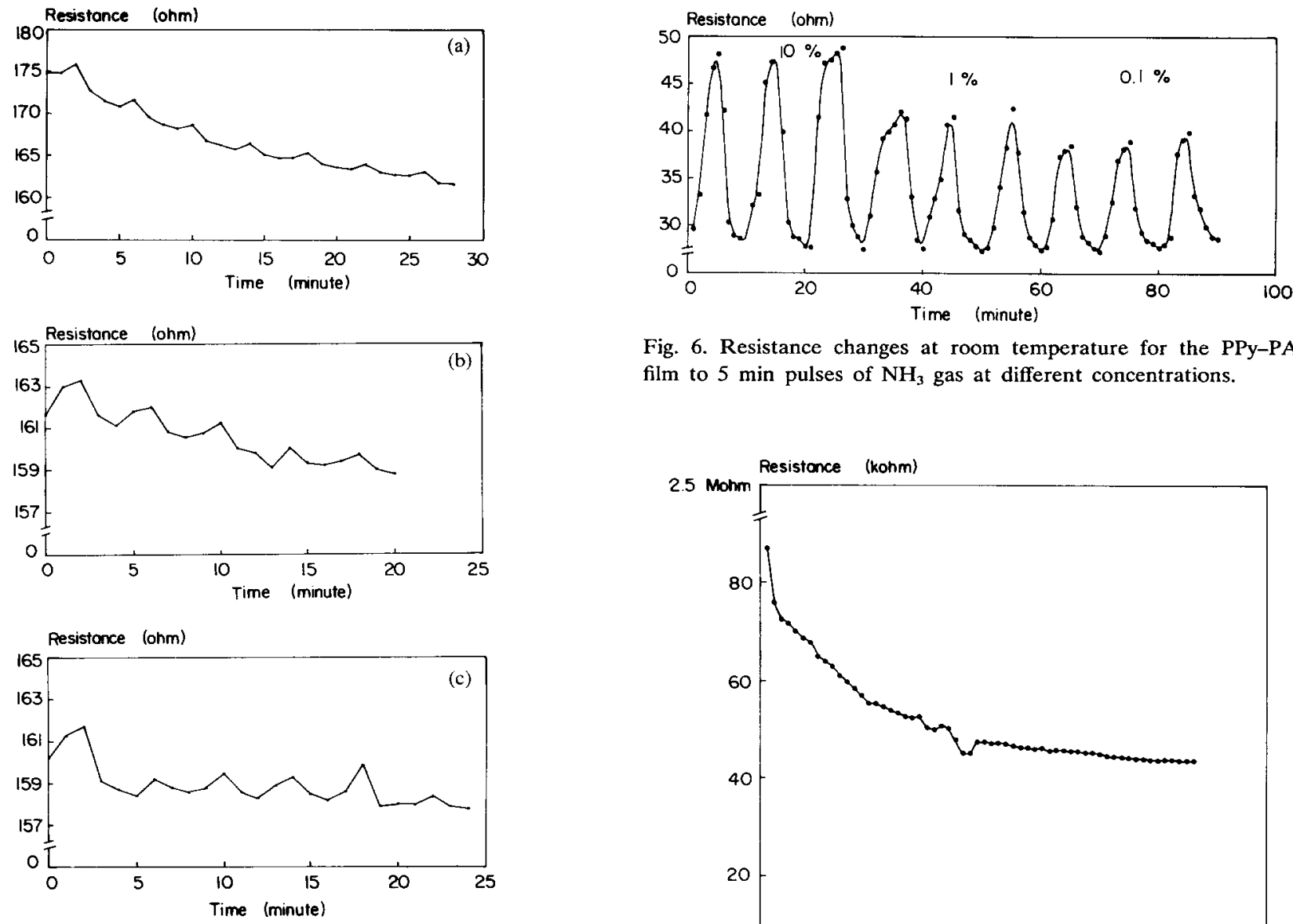

Fig. 4. Resistance changes at room temperature for the pure PPy film to 2 min pulses of (a) $10 \% \mathrm{NH}_{3}$, (b) $1 \% \mathrm{NH}_{3}$ and (c) $0.1 \%$ $\mathrm{NH}_{3}$. Flow rate, $150 \mathrm{ml} / \mathrm{min}$.
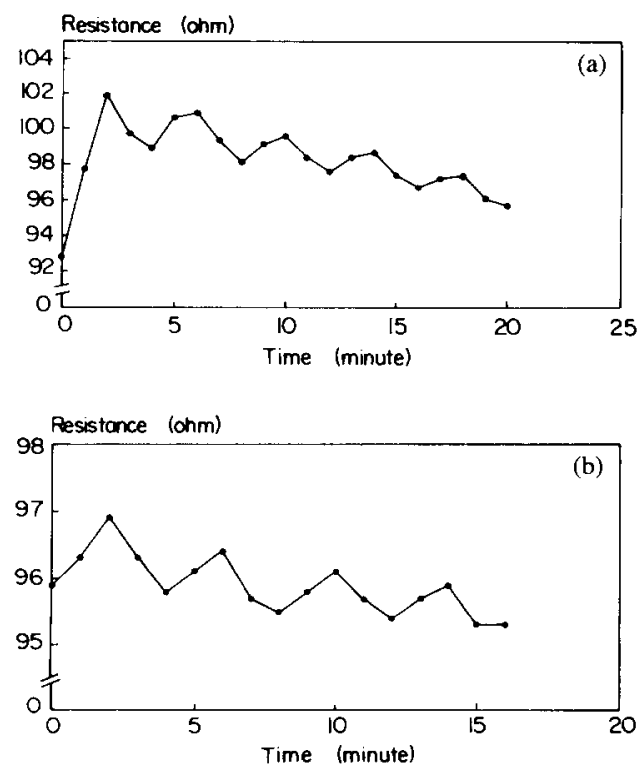

Fig. 5. Resistance changes at room temperature for the PPy-PA composite film to (a) $10 \% \mathrm{NH}_{3}$ and (b) $0.1 \% \mathrm{NH}_{3}$. Flow rate, 100 $\mathrm{ml} / \mathrm{min}$.

Fig. 6. Resistance changes at room temperature for the PPy-PA film to 5 min pulses of $\mathrm{NH}_{3}$ gas at different concentrations.

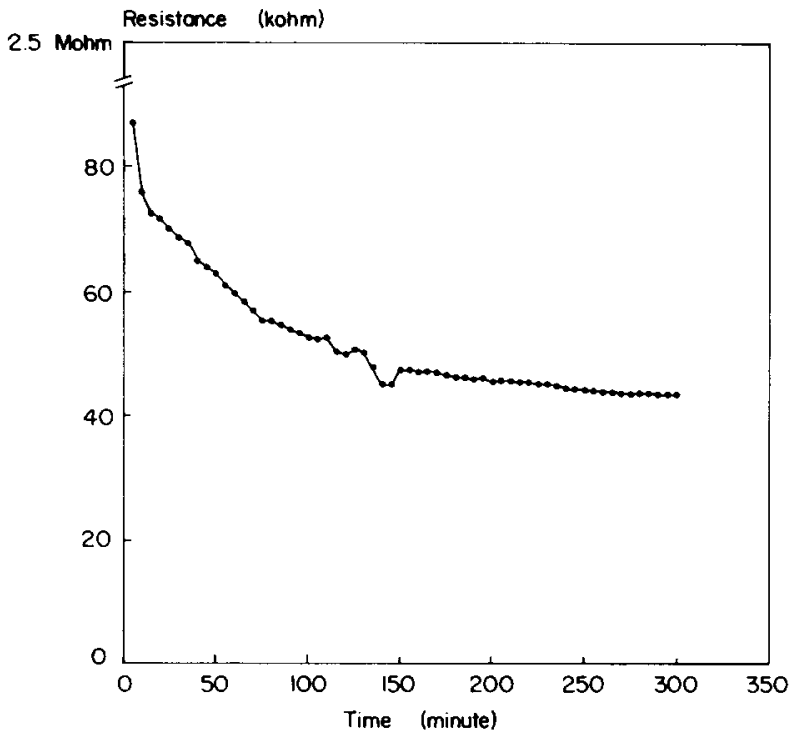

Fig. 7. Resistance changes at room temperature for a conducting pure PPy film sensor to $\mathrm{CO}_{2}$. Flow rate, $60 \mathrm{ml} / \mathrm{min}$.

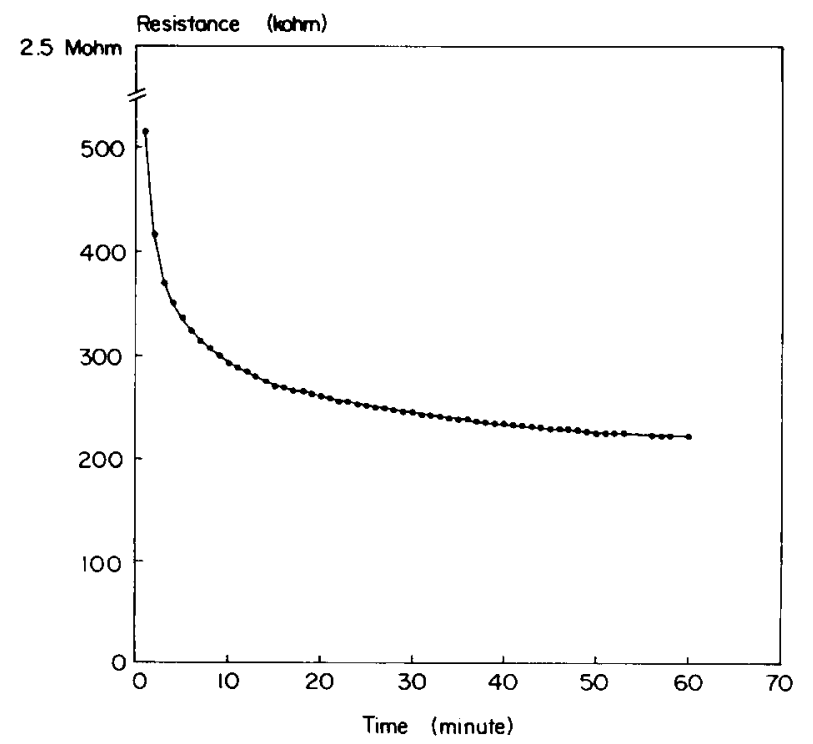

Fig. 8. Resistance changes at room temperature for a conducting PPy-PA film sensor to $\mathrm{CO}_{2}$. Flow rate, $60 \mathrm{ml} / \mathrm{min}$. 
monomer concentration was $0.01 \mathrm{M}$. The reference electrode was $\mathrm{Ag}^{0} / \mathrm{Ag}^{+}$. The thickness (about $50 \mu \mathrm{m}$ ) and the morphology of the films were discussed in a previous paper [19].

Cyclic voltammetry measurements were carried out in a $\mathrm{CH}_{3} \mathrm{CN}-0.1 \mathrm{M}$ TBAFB solvent electrolyte system. The reference electrode was $\mathrm{Ag}^{0} / \mathrm{Ag}^{+}$and the monomer concentration was $10^{-3} \mathrm{M}$.

The gas-sensing system consists of a measuring compartment where the film is connected to a digital multimeter, traps for drying the gas, a diluter where dry nitrogen is used as the diluent and measurement

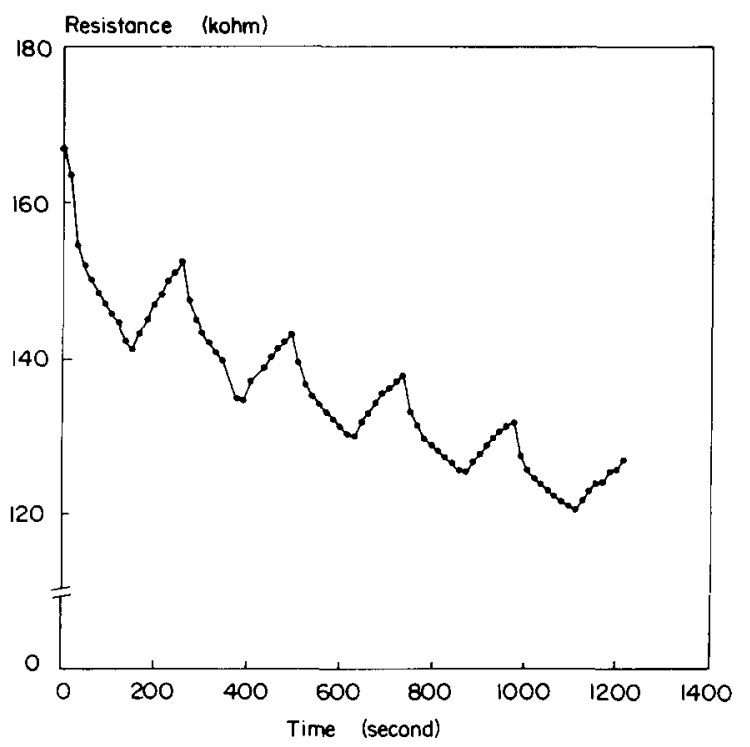

Fig. 9. Resistance changes at room temperature for a conducting pure PPy film sensor to $2 \mathrm{~min}$ pulses of $\mathrm{CO}_{2}$. Flow rate, $60 \mathrm{ml} / \mathrm{min}$.

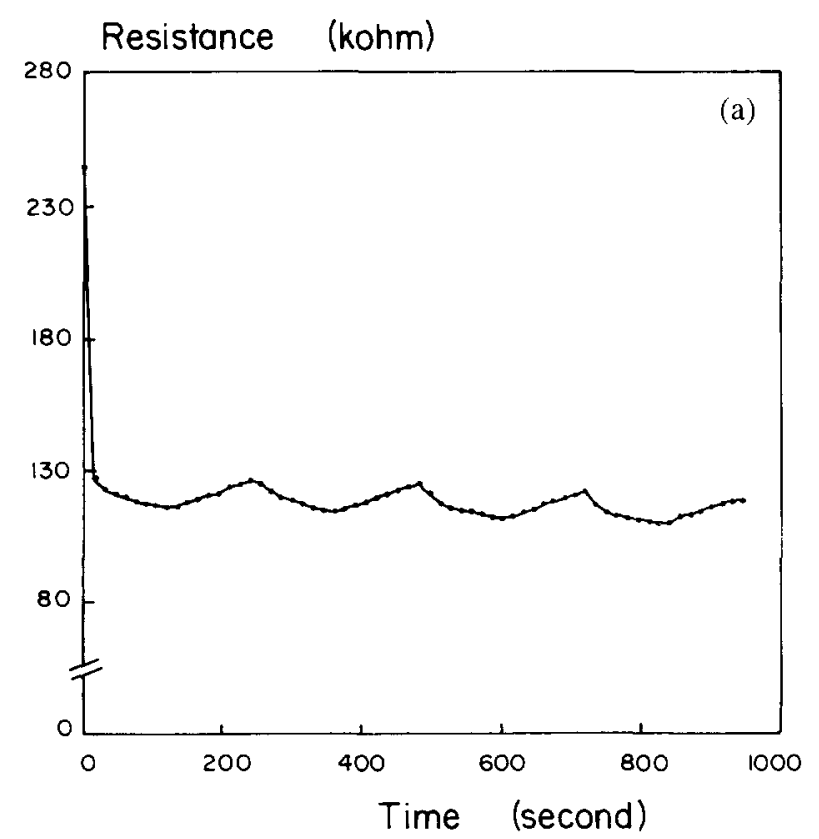

Fig. 10.
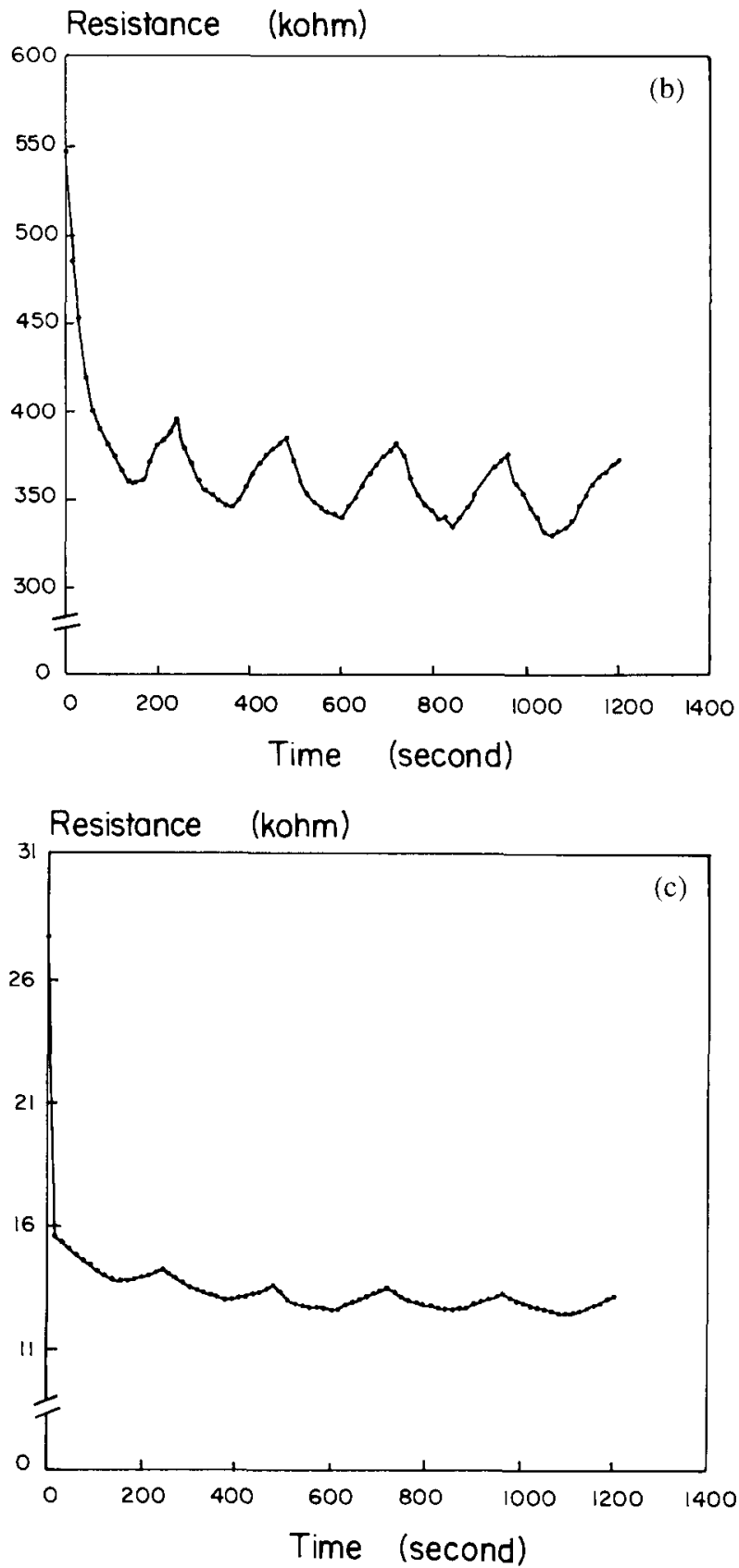

Fig. 10. Resistance changes at room temperature for a conducting PPy-PA film sensor to $2 \mathrm{~min}$ pulses of $\mathrm{CO}_{2}$. Flow rate, $60 \mathrm{ml} / \mathrm{min}$.

compartment for the carrier gas and flow rate [13]. Exposure to $\mathrm{CO}_{2}$ and $\mathrm{NH}_{3}$ gases was done under dry nitrogen gas and the system was opened to air when the passage of the gas ceased. The gas pulses ( 2 or 5 min) can be repeated many times in the case of electrolytic film and the real value cannot be given since it takes a considerable amount of time in order to be specific.

The experimental set-up for spectro-pyrolitic analyses consists of a quadrupole mass spectrometer, a high vacuum system, FT-IR and a sampling system. The mass spectrometer (Finnigan MAT MOdel 300 Quad- 

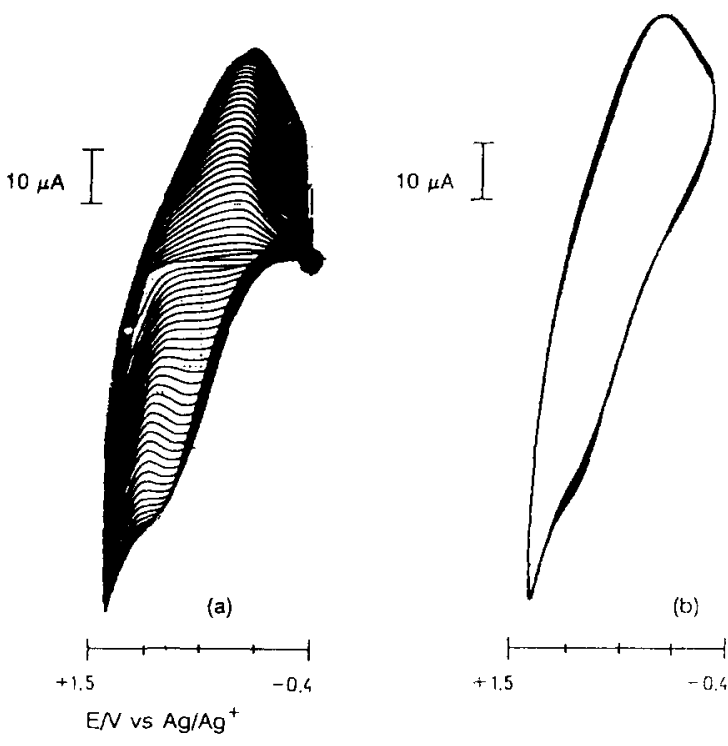

(b)

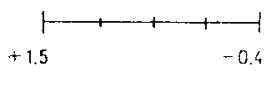

Fig. 11. Multisweep cyclic voltammograms of PPy (a) in $10^{-3} \mathrm{M}$ Py and $0.1 \mathrm{M}$ TBAFB- $\mathrm{CH}_{3} \mathrm{CN}$ solution and (b) last ten runs. Voltage scan rate, $900 \mathrm{mV} / \mathrm{s}$.

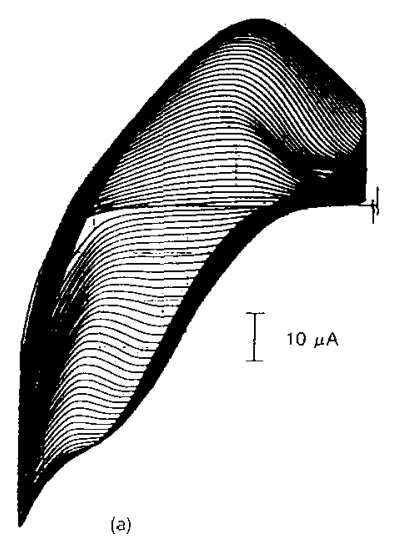

(a)

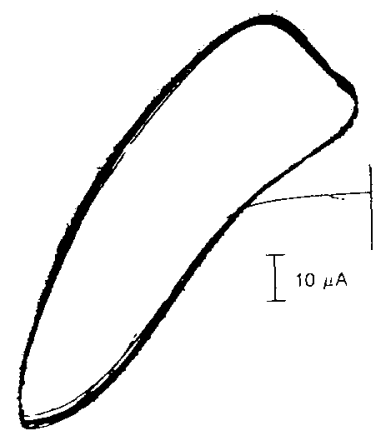

(b)

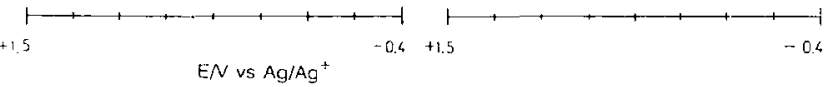

Fig. 12. Multisweep cyclic voltammograms of $\mathrm{Py}$ on a $\mathrm{Pt}$ electrode (a) in $10^{-3}$ Py and 0.1 M TBAFB- $\mathrm{CH}_{3} \mathrm{CN}$ and (b) no Py in medium. Voltage scan rate, $900 \mathrm{mV} / \mathrm{s}$.

rupole) has a control unit, analyser, vacuum and detection system, which is controlled by a PC. A singlebeam Nicolet Model 510 FT-IR spectrometer was used in these studies. The evolved gas analyses (EGAs) were carried out in a pyrolysis chamber and a furnace. The pyrolysis chamber consists of a glass tube which is connected to the mass spectrometer inlet system by a valve.

\section{Results and discussion}

It is known that $\mathrm{NH}_{3}$ is an electron-donating molecule that causes a reduction in the charge carrier density of PPy. The saturation points for pure PPy and composite

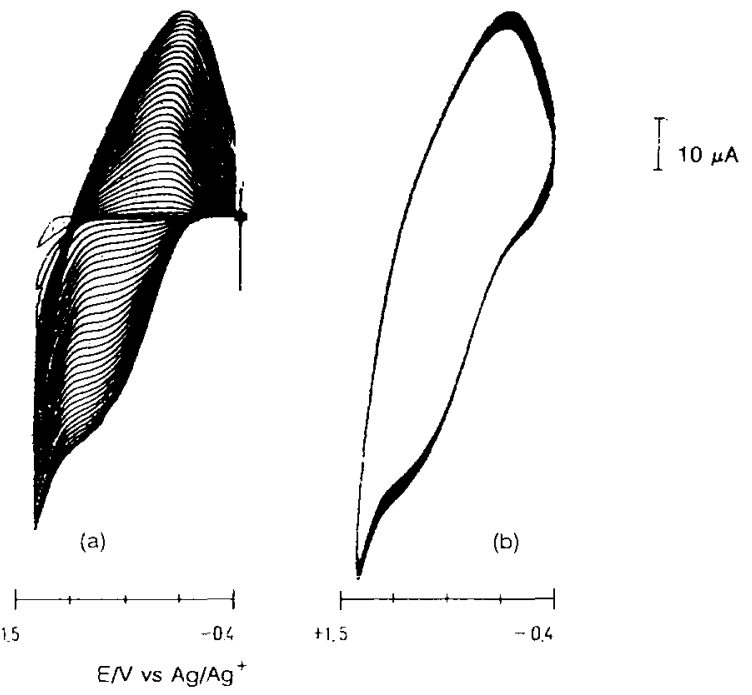

Fig. 13. Cyclic voltammograms for Py at the Pt-PA electrode (a) in $10^{-3} \mathrm{M}$ Py and $0.1 \mathrm{M}$ TBAFB- $\mathrm{CH}_{3} \mathrm{CN}$, and (b) last ten runs. Voltage scan rate, $900 \mathrm{mV} / \mathrm{s}$.

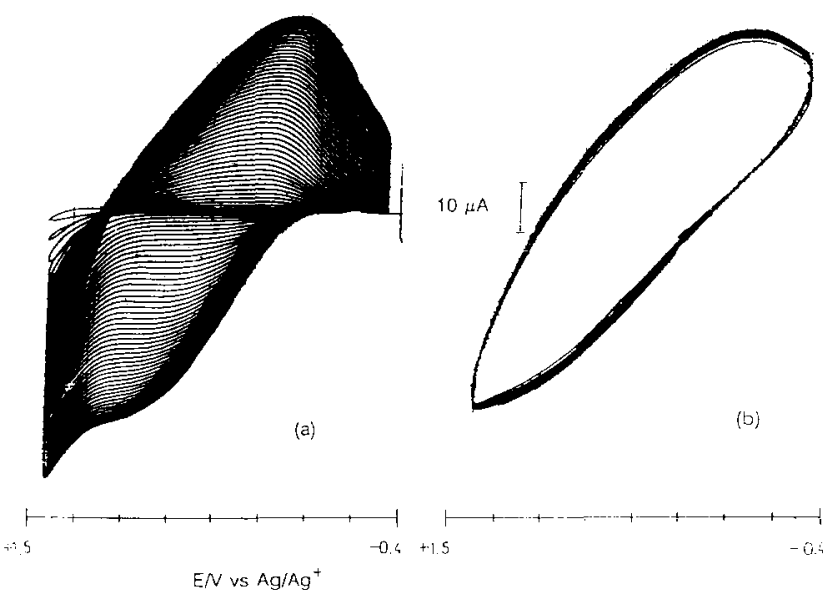

Fig. 14. Cyclic voltammograms of the PPy-PA electrode (a) in 0.1 $\mathrm{M}$ TBAFB- $\mathrm{CH}_{3} \mathrm{CN}\left(10^{-3} \mathrm{M} \mathrm{Py}\right)$ and (b) no Py. Voltage scan rate, $900 \mathrm{mV} / \mathrm{s}$.

films in the case of exposure to $\mathrm{NH}_{3}$ are given in Figs. 1 and 2, respectively. There is an increase in resistance when the films are exposed to $\mathrm{NH}_{3}$. Approximately 55 and $20 \mathrm{~min}$, respectively, are required to observe fivefold increase in the resistance for pure PPy and composite films. The time required to reach a saturation point decreases as the PA content increases, as shown in Fig. 3. This observation reveals the importance of having grafted composition with respect to the total amount of the two polymers.

As given in Fig. 4, the background resistance decreases as the number of pulses increases. The decrease is less at low concentrations of $\mathrm{NH}_{3}$, e.g., $0.1 \% \mathrm{NH}_{3}$. This behaviour can be attributed to the fact that the effect of $\mathrm{NH}_{3}$ gas $(2 \mathrm{~min})$ is not the same with the effect of air/moisture $(2 \mathrm{~min})$, yielding asymmetric triangles as the pulses are repeated. However, the electrolytic film 


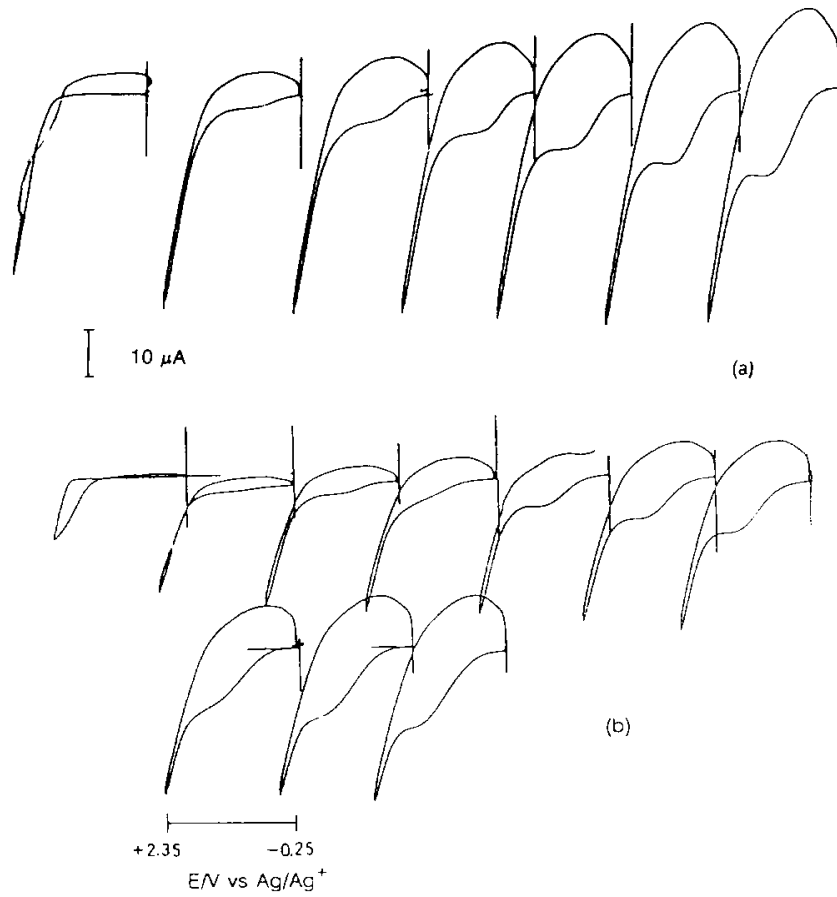

Fig. 15. Consecutive cyclic voltammograms of $\mathrm{Py}$ in $10^{-3} \mathrm{M}$ Py and $0.1 \mathrm{M}$ TBAFB- $\mathrm{CH}_{3} \mathrm{CN}$ (a) at a bare Pt and (b) at a PA-Pt electrode. Voltage scan rate, $900 \mathrm{mV} / \mathrm{s}$.

(PPy-PA) represents a different feature in terms of resistance changes.

Fig. 5 shows the response of PPy-PA composite films to $\mathrm{NH}_{3}$. The composite films show rapid and reversible responses compared to pure PPy film. The background resistance remains almost constant, indicating that the doping and undoping processes are very rapid and reversible. This behaviour supports the argument that the PPy-PA film is a different species compared to a simple mixture of the two polymers. This type of sensitivity enhancement was also observed in a previous study [13].

The lower limit of response as a function of gas concentration has not yet been investigated, but it is a fact that the resistance change is clearly dependent on concentration, as shown in Fig. 6 .

The response of PPy and composite films to $\mathrm{CO}_{2}$ was investigated. In this case the resistance decreases while it is exposed to the gas and increases when the film is exposed to air. Figs. 7 and 8 show the sensitivity of pure PPy and composite films respectively, to $\mathrm{CO}_{2}$, in order to observe the saturation point. For composite film, $50 \mathrm{~min}$ is required to reach saturation. For same amount of decrease in resistance, in the case of PPy, more than five times this period is required. Both pure PPy and composite films were saturated with $\mathrm{NH}_{3}$ prior to the $\mathrm{CO}_{2}$-sensing experiment. We exposed the film to $\mathrm{CO}_{2}$ for $2 \mathrm{~min}$ and found that the responses to repeated exposure to $\mathrm{CO}_{2}$ and then to air are reproducible. The response of pure PPy when exposed to
$\mathrm{CO}_{2}$ is shown in Fig. 9. The background resistance falls linearly as the number of pulses increases. Under the same conditions, the sensitivity of PPy-PA film shows similar behaviour upon exposure to $\mathrm{CO}_{2}$, as shown in Fig. 10. The background resistances are almost constant, implying that the doping and undoping processes are very rapid and reversible. Not only the background resistance but also the peak heights and widths (response time) remain constant throughout the measurement.

The electrochemical behaviour of PPy and PPy-PA composite film were recorded. Fig. 11 shows the cyclic voltammogram of PPy at a bare Pt electrode. Multisweep cyclic voltammograms were performed with a potential scan from -0.4 to $+1.5 \mathrm{~V}$. The plot shows a peak for oxidation of the film at around $+0.7 \mathrm{~V}$ on the anodic sweep and the corresponding reduction peak at around $+0.2 \mathrm{~V}$ in the cathodic sweep. The anodic peak potential of the polymer is almost linearly shifted towards the anodic direction with repeated cyclings. The anodic peak potential, which is $+0.7 \mathrm{~V}$ for the first runs, shifts to $+0.9 \mathrm{~V}$. As the runs are continued the electroactivity decreases, as shown in Fig. 11(b).

Further experiments were carried out in a medium where the monomer was not present. The cyclic voltammograms were run with a potential scan from -0.4 to $+1.5 \mathrm{~V}$, as shown in Fig. 12. The peak for oxidation shifts from +0.7 to $1.1 \mathrm{~V}$ and for reduction this shift is observed at $+0.1 \mathrm{~V}$ and, as shown in Fig. 12(b), the elecroactivity decreases resembling cyclic voltammograms taken in a medium where Py is present.

The PPy-PA electrodes were prepared by polymerizing Py using a PA-precoated Pt electrode [19]. The PA is sufficiently porous to allow Py and the electrolyte to diffuse across the film and react at the Pt electrode.

Multisweep cyclic voltammograms of PPy-PA composite electrodes are given in Fig. 13. The multisweep runs between -0.4 and $+1.5 \mathrm{~V}$ reveal an oxidation peak at $+0.7 \mathrm{~V}$. In the reduction region the peak is at $+0.2 \mathrm{~V}$. There is a shift during the cycling resembling PPy cyclic voltammograms. As seen from Figs. 11 and 13, the voltammograms of PPy-PA composite electrodes are qualitatively similar to Py voltammograms at bare $\mathrm{Pt}$, which indicates that both electrolyte and Py can permeate the PA-precoated Pt electrode. The last runs also resemble that of PPy and electroactivity decreases.

PPy-PA electrode is electroactive and the switching reaction is slower than PPy formed on a bare $\mathrm{Pt}$ electrode. The cyclic voltammograms of the PPy-PA electrode mounted in an electrochemical cell containing $\mathrm{CH}_{3} \mathrm{CN}-\mathrm{TBAFB}$ only are given in Fig. 14 . The cathodic peak potential shifts from +0.35 to $-0.225 \mathrm{~V}$ and electroactivity is lost, as shown in Fig. 14(b).

In Fig. 15 the two cyclic voltammograms with $\mathrm{PA}-\mathrm{Pt}$ and $\mathrm{Pt}$ as a working electrode are compared. At the Pt electrode, Py starts to polymerize with a sharp oxidation peak, while at the PA-Pt electrode it 


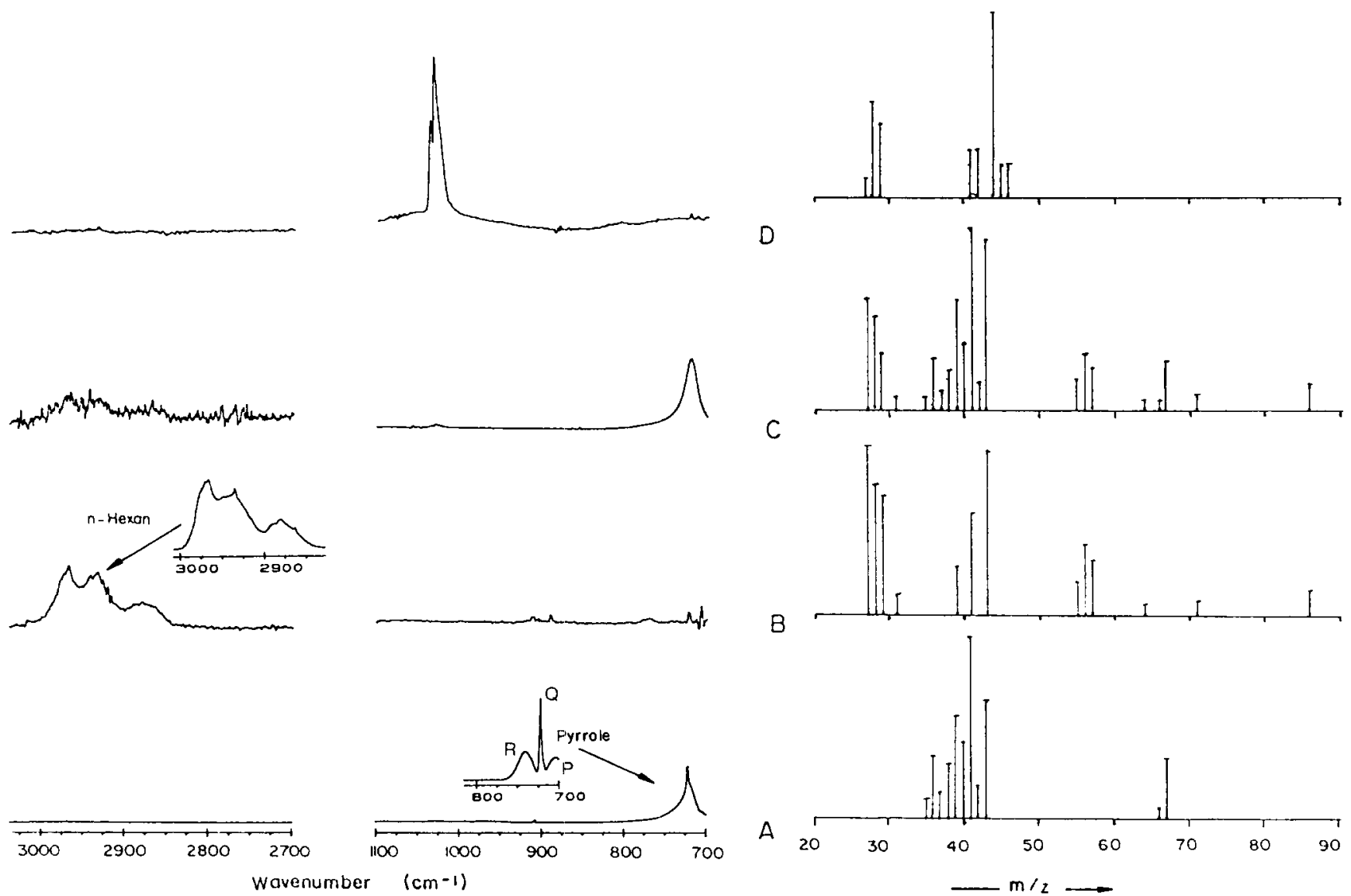

Fig. 16. FT-IR and mass spectra for (A) PPy, (B) PA, (C) mechanical mixture of (A) and (B) and (D) electrolytic film.
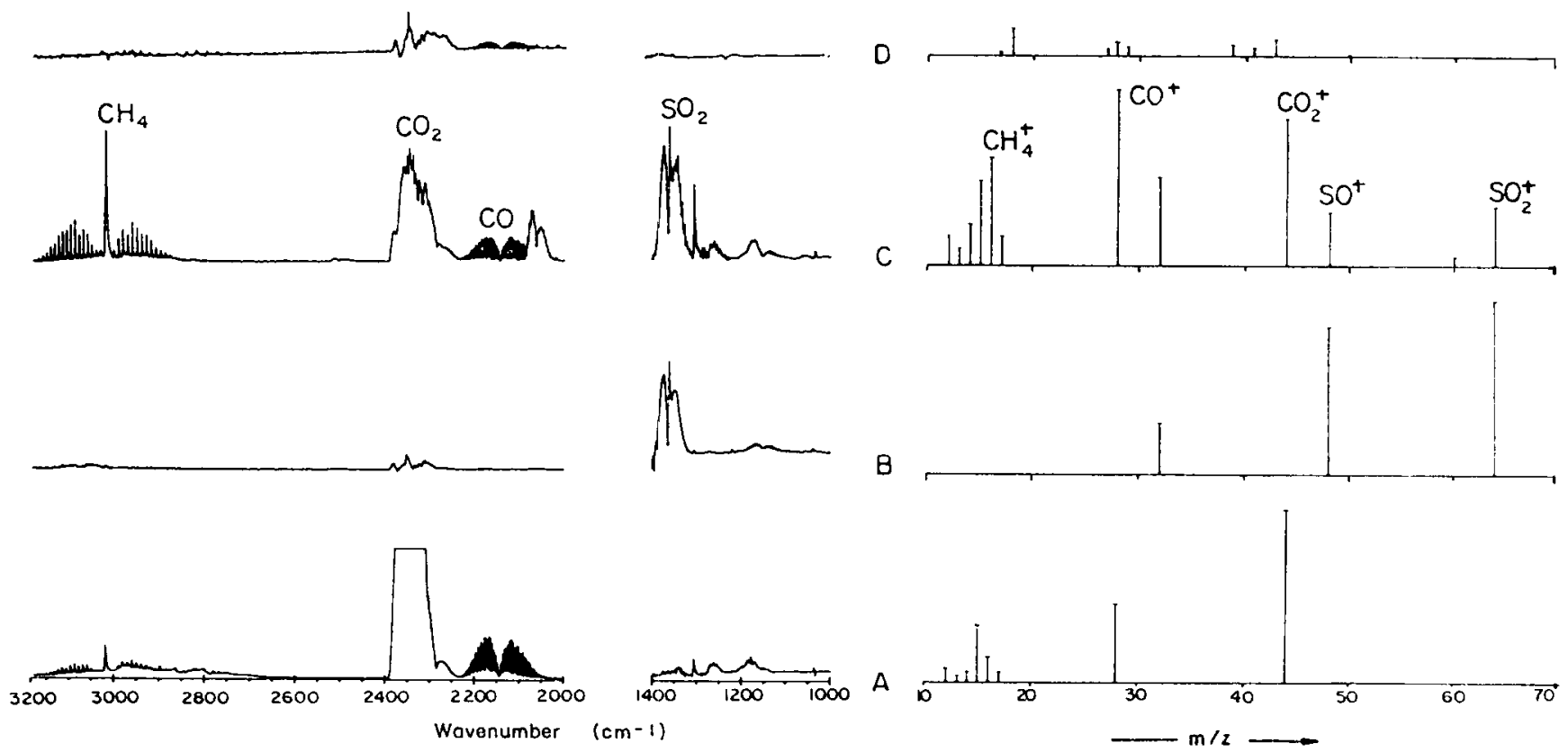

Fig. 17. FT-IR and mass spectra of (A) polybisphenol A carbonate, (B) PAn, (C) mechanical mixture of (A) and (B), and (D) electrolytic film [13]. 
polymerizes with a broader oxidation peak. The peak current in the former is stronger than in the latter. These differences may result from the fact that before the electropolymerization at the PA-Pt electrode, Py and the dopants $\left(\mathrm{BF}_{4}{ }^{-}\right)$have to diffuse onto the surface of the Pt electrode through the PA layer and then they have to rediffuse to the PA layer's outer surface to the electrolyte solution. Since the diffusion through the PA layer is inhibited to a greater or lesser extent, the electropolymerization of Py on the PA/Pt electrode is more difficult than directly at the Pt electrode. This may also be affected by the thickness of PA coated on the $\mathrm{Pt}$ electrode.

In Fig. 16, IR and mass spectra of pure PPy (A), pure PA (B), a simple mechanical mixture of the two polymers (C) and the electrolytic film (D) are given. The pyrolysis product of $P$ Py decomposes to its monomer and n-hexane. Both mass and FT-IR data support this result. In Fig. 16(A), P,Q,R branches until $280^{\circ} \mathrm{C}$ are same for the Py monomer (small figure) although it appears as a single peak in the spectrum since Fig. 16 represents spectra taken at $300{ }^{\circ} \mathrm{C}$. This change may be due to some heating effects. The peaks at 67 in the mass spectra are related to the mother peak of Py. The peak at 86 is due to n-hexane and the 27,28 , $29,39,41$ and 43 peaks are the fragments of n-hexane.

The peaks between 3000 and $2850 \mathrm{~cm}^{-1}$ in the IR stand for pure n-hexane (Fig. 17).

Pyrolysis products of the mechanical mixture of the two polymers give the same features as the pure PPy and PA. On the other hand, the electrolytic film yields completely different spectra indicating a possible chemical interaction between the two polymers, although the peak in Fig. 16(D) has not been identified yet. This possibility was discussed in terms of several parameters in a previous paper [19]. As to the pyrolysis products of PC-PAn [13], the pure PC decomposes to $\mathrm{CO}$ and $\mathrm{CO}_{2}$, while PAn decomposes into $\mathrm{SO}_{2}$, most probably due to its $\mathrm{SO}_{4}$ dopant (Fig. 17). The FT-IR spectra given in the previous study [6] together with the mass data reveal that the electrolytic film contains chemical linkages between PC and PAn in addition to its composite character. Fig. 17(D) represents the electrolytic film made up of $\mathrm{PC}$ and $\mathrm{Cl}^{-}$-doped PAn and explains the absence of $\mathrm{SO}_{4}$-related peaks.

Both composites (PC-PAn, PPy-PA) yield different features in IR and mass studies compared to their simple mixtures. These results support previous papers $[6,19]$ related to these new conducting polymers.

\section{Conclusions}

The gas sensitivity of the resulting material PPy-PA to $\mathrm{NH}_{3}$ and $\mathrm{CO}_{2}$ is better than pristine conducting polymer in terms of both rapid and reversible response. In a previous work [19] it was argued that the electrochemical blending of PPy with PA possibly yields a new species where a chemical bonding between the two polymers is very likely. The differences between the film and the mechanical mixture of the relevant polymers in terms of FT-IR, SEM and solubility studies led us to check the argument via pyrolysis and gassensing experiments. We believe this work confirms that electrolytic preparation of conducting polymers, such as PAn and PPy on polybisphenol A carbonate or PA-coated Pt electrode, yields new (possibly graft) conducting polymers contrary to the chemical blending procedure.

\section{Acknowledgements}

This work is supported by several organizations including the VW Foundation (I-67/069), METU Research Fund (94-01-03-04) and TUBITAK TBAG $\mathrm{U} 15 / 7$.

\section{References}

[1] C.K. Chiang, C.R. Fincher, Jr., Y. W. Paik, A.J. Heeger, H Shirakawa, E.J. Louis, S.C. Gau and A.G. MacDiarmid, Phys. Rev. Lett., 39 (1977) 1098.

[2] W.P. Su, J.R. Schieffer and A.J. Heeger, Phys. Rev. B, 22 (1980) 2099.

[3] A.F. Diaz, K.K. Kanazawa and G.P. Gardini, J. Chem. Soc., Chem. Commun., (1979) 635.

[4] F. Carmer, G. Tourillon, M. Gazard and J.C. Dubois, J. Electroanal. Chem., 148 (1983) 299.

[5] A.G. MacDiarmid, S. Mu, N.L.D. Somasiri and W. Wu, Mol. Cryst. Liq. Cryst., 121 (1985) 187.

[6] S. Doğan, L. Toppare and U. Akbulut, Synth. Met., 53 (1992) 29.

[7] T. Osaka, K. Naoi, S. Ogano and S. Nakamura, J Electrochem. Soc., 134 (1987) 2076.

[8] A. Mohammadi, O. Inganäs and I. Lundström, J. Electrochem. Soc., 133 (1986) 947.

[9] T. Yeu, K.M. Yin, J. Carbajal and R.E. While, J. Electrochem. Soc., 138 (1991) 2869.

[10] R. Noufi, D. Tench and L.F. Waren, J. Electrochem. Soc., 127 (1980) 1625.

[11] T. Skotheim, O. Inganäs, J. Prejza and I. Lundström, Mol. Cryst. Liq. Cryst., 83 (1982) 329.

[12] A.F. Diaz, I.I, Castillo, J.A. Logan and W. Lee, J. Electroanal. Chem., 129 (1981) 115.

[13] S. Doğan, L. Toppare, T. Yalçin, S. Süzer and U. Akbulut, Synth. Met., 60 (1993) 27.

[14] J.J. Miasik, A. Hooper and B.C. Tofield, J. Chem. Soc., Faraday Trans. 1, 82 (1986) 117.

[15] P.N. Bartlett, P.B.M. Archer and S.K. Ling-Chung, Sensors and Actuators, 19 (1989) 125.

[16] P.N. Bartlett and S.K. Ling-Chung, Sensors and Actuators, 19 (1989) 141.

[17] J.M. Slater and E.J. Watt, Anal. Proc., 26 (1989) 397.

[18] M. Sato, S. Tanaka and K. Kaeriyama, J. Chem. Soc., Chem. Commun., (1989) 713.

[19] F. Selampinar, U. Akbulut, T. Yalçin, S. Süzer and L. Toppare, Synth. Met., 62 (1994) 201. 